$\overline{\text { ReVIEW ARTICLE }}$

\title{
INTRICACIES OF IDENTITY
}

\author{
CHRISTOPHER TANCREDI \\ Yokohama National University
}

Indices and Identity, by Robert Fiengo and Robert May, MIT Press, Cambridge, MA, 1994, xvii $+315 p$.

\section{Introduction}

Fiengo and May (1994) (henceforth F\&M) propose an analysis of restrictions that obtain on when two referring expressions can stand in a certain type of identity relation. Various terms have been employed in the literature to describe the identity relation in question, including "coreference," "intended coreference," "(anaphoric) dependence," "antecedence," and "common reference" among others. The exact nature of this relation has been a question of some debate. Some authors (e.g. Chomsky (1976), Lasnik (1976)) have argued that it is purely a pragmatic relation, i.e., that the grammar of English, for example, never determines that any two terms stand in this relation. Under such analyses, the basic function of pronouns and names is to enable a speaker to refer to people, objects, etc., the only restriction imposed by the grammar on this function being that of enforcing certain pairs of expressions to be used to refer to distinct objects. $F \& M$ argue in opposition to this view that two expressions can be determined by the grammar to co-refer, following in this respect Chomsky (1981), Evans (1977, 1980), Higginbotham (1983), and Reinhart (1983). On F\&M's view, restrictions on reference are indirect, mediated by restrictions on syntactic representations. Two referring expressions syntactically identified must co-refer, though whether other pairs of expressions co-refer depends only on the intentions of the speaker and the circumstances of utterance.

Tied up with the question of when two expressions should be determined by the grammar to stand in some identity relation is the 
question of how this relation should be represented. Mechanisms that have been proposed to capture this relation include symmetric representation systems such as the indexing system of Chomsky (1981) as well as inherently asymmetric systems such as the linking representation of Higginbotham (1983). F\&M argue for a single Chomsky-like symmetric identity relation, but superimpose on this a Higginbothamlike asymmetric sentence-internal antecedence relation.

In this paper, I will examine the problem of constraining identity from the perspective of when two expressions can and when they cannot be used with the implicit intention that they be interpreted in an identical fashion. Whenever two expressions are appropriately used with such an intention, I will say that they stand in a relation of intended identity. The question I pose here is whether it is possible to reduce intended identity to syntactic identity. I argue that such a reduction is impossible if the grammar contains only a single means of syntactically representing identity as do all of the analyses referred to above, but is possible if the grammar contains two distinct such representations.

\section{Fiengo and May's approach}

F\&M are very explicit in the relations they take to hold between syntactic assignments of indices and relations of coreference. For them, coindexing determines coreference (or more accurately covaluation), while non-coindexing fails to determine - though it does permitcoreference. They formalize their analysis as follows. The interpretation of an indexed expression is determined by the value of the index and a sequence of individuals $\sigma$. In particular, the value of an expression $x_{i}$ is the individual assigned to the $\mathrm{i}^{\text {th }}$ position of $\sigma$. Any two expressions bearing occurrences of the same index automatically - by the grammatical mechanism for interpreting indexed expressions-pick out the same individual. Two expressions bearing occurrences of distinct indices, in contrast, will refer to individuals via different positions in $\sigma$. Since $F \& M$ allow different positions in a sequence to be assigned the same individual, it is possible for non-coindexed expressions to corefer. However, coreference in this case is merely an option that is left open by the grammar, not one forced by the grammar. This gives rise to the three-way distinction illustrated below, where $\mathrm{a}, \mathrm{b}$, and $\mathrm{c}$ are taken to be distinct individuals. 

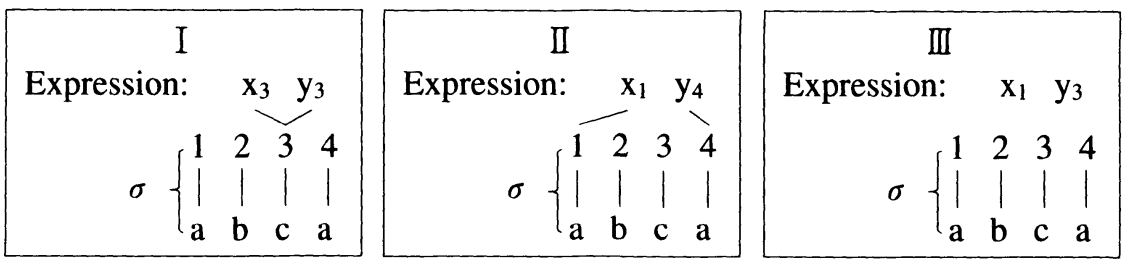

The connection between the three cases illustrated in I-III above and the intentions with which people utter various sentences are not laid out explicitly, though it is possible to reconstruct F\&M's intentions from the arguments that they give. A speaker can employ two coindexed expressions only when she intends to refer to the same individual twice. It is also possible to intend to refer twice to the same person using non-coindexed expressions, though this is never forced by the syntactic principle interpreting indices alone. The need to allow for this possibility comes most directly from the contingency of identity statements. In the sentence in (1) below, if he were coindexed with John, the sentence would under F\&M's analysis be a tautology, on a par with John is himself.

(1) He is John

Since (1) is not a tautology under any interpretation, it follows that he and John must be non-coindexed, a consequence that follows independently for $\mathrm{F} \& \mathrm{M}$ from Binding Theory Condition C. However, assertion of this sentence commits the speaker to he and John being the same individual, and hence it is not plausible to assume that the sentence can be asserted without intending identity between these two expressions. Therefore, non-coindexing must not entail lack of intended identity: the situation depicted in II above must be allowed.

While intended identity is clearly required for identity statements such as (1), allowing for intended identity under non-coindexing threatens to render vacuous any theory of binding. In (2), the fact that coindexing of he and John violates Condition C should have no effect on the possibility of intending the two expressions identically.

(2) He likes John

Just as with the identity statement in (1), intended identity should be derivable in (2) from a representation parallel to II above. As we will see below, the availability of such an interpretation for (2) is sensitive to the context in which it occurs. Context initially, however, such an interpretation is unavailable, in contrast to (1). F\&M account for this 
fact essentially by taking non-coindexing to give rise to an absence of intended identity by default unless there is an overriding pragmatic or semantic reason to posit intended identity. In (1), the semantics of the copula is provides such a reason. In (2), however, nothing does. This gives a needed account of the distinction between (1) and (2), but only by pushing the problem off to the side, since F\&M do not indicate what such overriding pragmatic or semantic reasons can be.

The lack of a clear proposal for deriving intended identity from representations lacking syntactic identity stands as a blemish on F\&M's analysis. Intuitions about the connections between sentences and the intentions with which they can be paired provide the core data that a theory of grammar is called upon to help explain. The need to account for these connections is especially clear in the realm of Binding Theory. It has been widely observed that in sentences such as (3) it is perfectly natural to intend all occurrences of the expressions John/he/ him identically (and to be taken to so intend), while in (4) it is much less natural to do so. (Here and throughout, I indicate intended identity between two expressions by underlining those expressions.)

(3) a. John's mother loves John.

b. John's mother loves him.

c. John thinks Mary loves him.

(4) a. *John loves him.

b. * John loves John.

c. *He thinks Mary loves John.

An analysis which makes a syntactic distinction between the two classes of cases can potentially form the basis for explaining this contrast. However, it will only succeed in doing so in one of two circumstances: (i) the referential intentions with which a sentence can be uttered are completely determined by the syntactic representation of the sentence, or (ii) the cases in which intentions and syntactic representations fail to match up are completely accounted for by some other aspect of the theory of communication. F\&M's analysis is presumably intended as an analysis of the second sort, though in this respect it only provides half of the required explanation. I propose in contrast to construct a proposal of the first sort. 


\section{Single Identity Syntax}

In this section I sketch two broadly syntactic approaches to accounting for the data in (3) and (4): a symmetric approach to binding modeled after $\mathbf{F} \& \mathbf{M}$, and an asymmetric approach to binding modeled after Higginbotham (1983). I show that neither type of identity can alone form the basis for a reduction of intended identity to syntactic identity. I then develop an analysis admitting both types of syntactic identity which can account for the data.

\subsection{Symmetric Syntactic Identity}

Consider first a purely symmetric approach to binding such as that of F\&M. Using indices to represent a symmetric relation of syntactic identity, the distinction between the examples in (3) and those in (4) is accounted for by restricting the distribution of indexed expressions. Chomsky (1981) formulates such restrictions as Conditions B and C of the Binding Theory, given below and adopted by F\&M, where the class of R-expressions is taken to minimally include names.

( 5 ) Condition B: A pronominal is free in its binding category

Condition C: An R-expression is free.

An expression is free just in case it is not bound, where binding is defined as in (6). ${ }^{1}$

(6) $\alpha$ is bound by $\beta$ if and only if $\alpha$ and $\beta$ are coindexed and $\beta$ c-commands $\alpha$.

Assuming an appropriate characterization of the notion binding category, ${ }^{2}$ these conditions serve to rule ungrammatical the following representations for the sentences in (4) above.

(7) a. John loves himi $_{i}$.

b. John ${ }_{\mathrm{i}}$ loves $\mathrm{John}_{\mathrm{i}}$.

c. $\mathrm{He}_{\mathbf{i}}$ thinks Mary loves $\mathrm{John}_{\mathbf{i}}$.

If intended identity reduces to syntactic identity, the unacceptability of intended identity between the relevant occurrences of John/him/ he in (4) follows directly from the ungrammaticality of these representations. In (7a), the pronoun him is bound by John in its binding category ( $=$ the matrix sentence) in violation of Condition B. Similarly, in (7b)

1 I will only be concerned with A-binding throughout, and so take $\alpha$ and $\beta$ in (6) to be in A-positions.

2 For the purposes of this paper, it will suffice to define the binding category of an expression as the minimal NP or S containing that expression. 
and (7c), the object occurrence of the R-expression John is bound in violation of Condition $\mathrm{C}$.

While this analysis accounts for the simple cases considered in (4), phenomena known at least since Lakoff (1968) pose a serious problem when larger contexts are considered. (8) is taken from Evans (1980) (footnote 30), upon which (9) is modeled.

(8) What do you mean Oscar loves no one? He loves OSCAR.

(9) Although not many people like John, JOHN/HE likes John I him.

The problem these examples pose for F\&M's analysis is clear. In all of the examples in (8) and (9), it is possible to utter the italicized sentences in the contexts given intending identity between the subject and object NPs and to be readily understood as so intending. If the expressions in question are coindexed, then all of these examples should violate either Condition $B$ or Condition $C$, on a par with the parallel context initial sentences in (4).

F\&M recognize the problem such examples pose for their analysis, though not I believe in its full scope. To solve the problem, they analyze a subset of the examples adduced by Evans as belonging to a mathematical meta-fragment of English in which Binding Conditions do not apply. The existence of this meta-fragment they justify by the need for logical discourse, in which repetition of an expression serves a different purpose than use of a distinct coreferential expression in the logical structure of an argument. The examples in (8) and (9), however, are not of the type one typically encounters in logical discourse, and nor are they in any way restricted to use in logical or mathematical discourse. Their inclusion in the proposed metafragment would thus be mysterious at best, though excluding them from this meta-fragment leaves F\&M in need of an account of the acceptability of intended identity between the underlined expressions. If we reject F\&M's proposal, as I believe we should, then an alternative analysis of the facts is required.

The above problem can be circumvented by allowing an individual expression to bear more than one index, with each index independently determining the referent of the expression. In this way, identity between the two occurrences of John/he/him in (9) can be represented indirectly, each expression being directly coindexed with the occurrence of John from the preceding clause, but bearing a distinct index from that of the other occurrence of John/he/him in the same 
clause, as illustrated in (10) below for one instantiation of (9).

(10) Although not many people like $\underline{J o h n}_{1,2}, \underline{H E}_{1}$ likes him 2 .

Such an analysis is consistent with the view that syntax encodes identity symmetrically, and thus is well within the spirit if not the letter of F\&M's analysis. All that needs to be added to the analysis is a restriction that no singular expression can have multiple distinct referents. Singular expressions bearing occurrences of multiple indices would on this account be required to be interpreted via sequences that assign identical referents to each of their distinct indices, a requirement satisfied in IV but not in V.
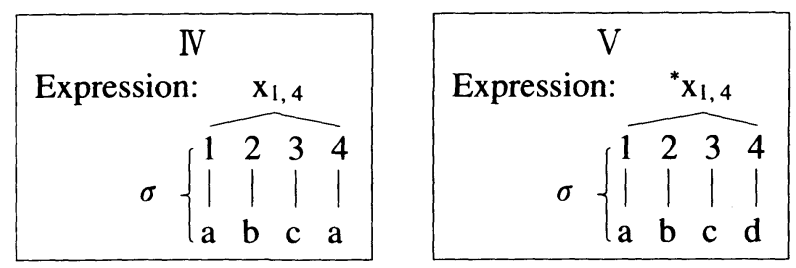

By continuing to treat syntactic restrictions on identity in terms of the actual index used, we could admit (10) as a representation in which he and him are determined to be intended identically without the one expression illicitly binding the other.

While a modification along the lines just sketched may well be called for, consideration of the example in (11) indicates that at the very least something more needs to be said.

(11) ${ }^{*}$ John said he likes him. ${ }^{3}$

(12) $\overline{\mathrm{John}}_{1,2}$ said he $\underline{\underline{1}}_{1}$ likes $\underline{\mathrm{him}}_{2}$.

The representation of (11) given in (12) is parallel to (10) above in that John/he/him are all determined by the syntax to be identical while satisfying the Binding Conditions in (5). This example is distinct from the previous examples considered, however, in that in (11), under a normal pronunciation in which neither pronoun bears a pitch accent, intended identity between the three expressions is unacceptable.

If the explanation given for the acceptability of (8) and (9) is to be maintained, a distinction must be found between these examples and (11) which makes it possible to analyze the former as acceptable and the latter as unacceptable. Noticing that (8) and (9) differ from (11)

3 This example is taken from Higginbotham (1983). 
in their focus properties, an explanation in terms of focus would appear plausible. This is supported by the observation that altering the focus structure of (11) can significantly improve the acceptability of that example, as illustrated in (13).

(13) a. (?)John said HE likes him.

b. John said he likes HIM.

Higginbotham (forthcoming) observes that this is a general phenomenon; whenever intended identity is possible between two expressions one of which would appear to violate Condition B or C of the Binding Theory, at least one of the two expressions must be focused. This observation is supported by the examples considered earlier, and can be substantiated even more forcefully with the examples in (14), where focus is indicated with CAPITALIZATION, and deaccenting (or what F\&M call down-stressing) with small italics.

(14) Although many people like John, JOHN $/ \mathrm{HE} /{ }^{*}{ }_{\text {John }} /{ }^{*}$ he HATES $_{\text {John }} /$ him.

Here, it is clear that focal properties play an important role in allowing intended identity between two expressions which might not otherwise allow of such an interpretation. Indeed, we can even exploit this effect to distinguish between an acceptable reading and an unacceptable reading of the examples given originally in $(4 a, b)$, as indicated in (15) below. ${ }^{4}$
a. JOHN LOVES HIM $/{ }^{*}{ }_{\text {him }}$.
b. JOHN LOVES JOHN $/ *_{\text {John }}$.

A first attempt to account for the effects of focus structure on intended identity within F\&M's analysis would be to exempt all focused expressions from participating in syntactic disjointness conditions. Intended identity between expressions could still be captured syntactically on such an account, though the disjointness conditions would be selectively blind to any such representations involving focused expressions. However, the following paradigm (constructed from (15a)) strongly militates against such a solution.

(16) a. ${ }^{*}$ JOHN HATES $\mathrm{him}$.

b. JOHN's MOTHER HATES him.

c. JOHN HATES himself. $_{\text {. }}$

In (16a) we have the exact same sentence with the exact same focus

${ }^{4}$ Here and throughout, examples are intended as context initial unless otherwise indicated. 
structure that we found to be felicitous under an intended identity reading in the context of (14). Context initially, however, the sentence gives rise to typical Condition B type effects - it is unacceptable to intend the subject and object to be identical. While one might be tempted to account for these facts by arguing that the focus structure itself is unacceptable context initially, consideration of the examples in $(16 \mathrm{~b}, \mathrm{c})$ makes such an approach implausible. These examples have the same essential focus structure as (16a) and yet are perfectly acceptable context initially with intended identity between John and $\operatorname{him}(\operatorname{self})$. If focused expressions simply failed to participate in disjointness conditions, the unacceptability of a context initial occurrence of (16a) under an intended identity reading would be unexplainable.

A second approach to consider is to concentrate not on the expressions which are focused but rather on those which are deaccented. I argued in Tancredi $(1992,1993)$ that a sentence containing a deaccented expression is only felicitous if the context contains another expression identical to the deaccented expression at the point at which this latter expression is processed. If we assume that two pronouns and/or R-expressions are identical for the purposes of deaccenting only if they each bear an occurrence of the same index, then such a constraint has the effect of forcing deaccented pronouns and Rexpressions to be coindexed with another such expression occurring previously in the context.

To illustrate how this analysis can combine with a syntactic analysis of identity to restrict intended identity, consider the contrast between the unacceptable example in (4a) and the acceptable example in (14c), both repeated in (17) with the relevant aspects of focus structure implicitly assumed earlier made explicit.

(17) a. *JOHN LOVES him.

b. Although many people like John, JOHN HATES him.

By hypothesis, deaccenting of the pronoun him is felicitous only if there is some expression in the context preceding the pronoun with which it is coindexed. Taking these examples to occur context initially, in (17a) there is only one expression which can possibly satisfy this requirement, the subject occurrence of John. In (17b), on the other hand, there are two separate expressions which can satisfy this requirement, the subject occurrence of John in the same clause as the pronoun, and the object occurrence of John in the subordinate clause. 
Partial representations of (17) which satisfy the identity requirement imposed by deaccenting of the pronoun are illustrated in (18).

(18) a. JOHN 1 LOVES him $_{1}$.

b. i. Although many people like JOHN, JOHN 1 HATES him $_{1}$.

ii. Although many people like $\mathrm{JOHN}_{1}$, JOHN HATES him 1 .

The representations in (18a) and in (18b-i) directly violate Binding Condition B as formulated in (5) above. Since (18a) is the sole representation available for (17a) which satisfies the requirement imposed by deaccenting of the pronoun, the unacceptability of (17a) follows directly. In the case of (17b), on the other hand, we have an additional, formally distinct way of satisfying the identity constraint imposed by deaccenting the pronoun, that represented in (18b-ii). Since it is possible under our current working assumption to coindex the two occurrences of John without thereby coindexing the second occurrence of John with the pronoun, it follows that all three expressions can be determined to be identical without violating Binding Condition B by representing (17b) as in (19).

(19) Although many people like $\mathrm{JOHN}_{1,2}, \mathrm{JOHN}_{2}$ HATES him 1 .

While the solution just sketched accounts for the difference in acceptability between $(17 \mathrm{a})(=(4 a))$ and $(17 \mathrm{~b})(=(14 \mathrm{c}))$, this analysis cannot be extended to account for the unacceptability of (11) above, repeated here as (20) with relevant aspects of focus structure made explicit.

(20) * John said $\underline{\text { he }}$ LIKES $\underline{\text { him }}$.

If we assume that the pronouns in (20) are all deaccented, then here as in (17) they will have to be coindexed with some preceding expression. However, this restriction can be satisfied in a way in which no Condition $\mathrm{B}$ violation results by assigning indices as in (21) below.

(21) $\operatorname{John}_{1,2}$ said he 2 likes him 1 .

Here, each of the deaccented pronouns is coindexed with a preceding expression (namely John) as required, but they are not coindexed with each other. Since separate coindexing of the pronouns with John does not violate Condition B, appeal to constraints imposed by deaccenting does nothing to help us account for the unacceptability of (20). Reducing intended identity to a symmetric analysis of syntactic identity like the extension of F\&M's analysis under consideration appears impossible. 


\subsection{Asymmetric Syntactic Identity}

Evans $(1977,1980)$ posited an asymmetric identity relation in order to get around the problems posed by examples like (8). This approach to syntactic identity is pursued further in Higginbotham (1983), upon which I base the present discussion. Taking the idea to its extreme, suppose we take such an asymmetric identity relation to be the only means available for representing identity syntactically. ${ }^{5}$ Whether intended identity can be reduced to syntactic identity will then depend upon whether it is possible to capture restrictions on intended identity such as those seen in (4), (11) etc. above while allowing for the exceptional cases in (8) and (9) employing only an asymmetric identity relation. Following Higginbotham (1983), I indicate this relation with a headed arrow extending from one expression (the dependent expression) to another (the antecedent expression). If intended identity is to be reduced to this asymmetric syntactic identity relation, then for two expressions to be intended identically they will have to be connected either directly or indirectly by links. ${ }^{6}$

To evaluate the feasibility of such an approach, consider first the (context initial) examples from (15), repeated here, where the sole factor distinguishing between the acceptable examples and the unacceptable ones is whether the object John/him is focused or deaccented.
a. JOHN LOVES HIM $/{ }^{*}{ }_{h i m}$.
b. JOHN LOVES JOHN $/ *_{\text {John }}$.

Here as above it is not possible to account for the contrast simply by excluding focused expressions from participating in conditions on disjointness, though it is possible to adopt the deaccenting approach sketched earlier. In order for the two occurrences of John/he/ him to be intended as identical, by hypothesis they must be connected by links. Assuming these sentences to occur context initially, we have exactly two possible representations for each that satisfy this condition, illustrated in (22) below.

5 Neither Evans nor Higginbotham adopt this extreme position. Though I too will conclude that the position cannot be maintained, I tentatively adopt it to explore the limitations of an asymmetric analysis of identity.

6 Higginbotham (1983) does not attempt to reduce intended identity to syntactic identity, and hence is not committed to such a restriction. Indeed, since Higginbotham allows two R-expressions to be intended identically but explicitly prohibits R-expressions from being linked to any other expression, he can be seen as rejecting the possibility of reducing intended identity to linking out of hand. 
(22)
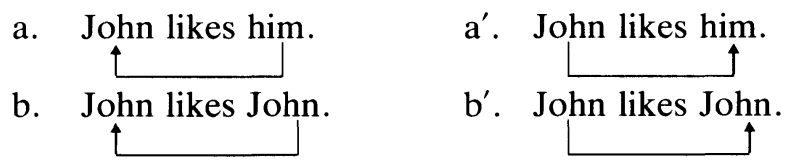

In order to account for the acceptability of the examples in (15) when the object is focused, at least one representation in each pair in (22) must be grammatical. We can account for the unacceptability of these same sentences when the object is deaccented by assuming that the other representation is ungrammatical, and by further assuming that deaccenting of the object is only compatible with the ungrammatical representation.

To formalize this analysis, assume that an R-expression or pronoun can be deaccented only if it is linked to another R-expression or pronoun in the preceding context. For the sentences in (15) containing a deaccented object NP, only the representations in $(22 a, b)$ will satisfy this restriction. We can then exclude these representations with a simple modification to the Binding Conditions from (5) (repeated here) by redefining the notion of bound (and hence also the notion of free) so as to make reference to linking rather than to coindexing, as in (23).

( 5 ) Condition B: A pronominal is free in its binding category. Condition C: An R-expression is free.

(23) $\alpha$ is bound by $\beta$ if and only if $\beta$ c-commands $\alpha$ and $\alpha$ is linked to $\beta$. $^{7}$

In each of the examples in $(22 a, b)$, some expression is illicitly bound under this revised interpretation of the Binding Conditions, him in (22a), and John in (22b), thus accounting for the unacceptability of the relevant examples from (15). Those cases in (15) in which both the matrix subject and the object are focused can satisfy the Binding Conditions since these can be represented as in $\left(22 a^{\prime}, b^{\prime}\right)$, where no such illicit binding relations occur.

With the above analysis, we can give an account of the contrast

7 To simplify discussion, I assume following Higginbotham that linking is a transitive relation, so that if $a$ is linked to $b$ and $b$ is linked to $c, a$ thereby counts as linked to $c$. This assumption will preclude a third logical potential representation for (9d) in which him is linked to John and John is linked to he, since by transitivity of linking him would be linked to he in violation of Binding Condition B. I will also assume that expressions can be directly linked to at most one other expression. Again this assumption is not a necessary one, though it helps to simplify definitions and discussion. 
found earlier between the acceptable examples in (8) and (9) and the unacceptable example in (11). In order for any of these examples to be grammatical, it must be possible to represent them in such a way that (i) every deaccented expression contained within the example is linked to some formally preceding expression, (ii) each occurrence of John/he/him is connected by links (directly or indirectly) with the others, (iii) no occurrence of he/ him is bound in its binding domain, and (iv) no occurrence of John is bound at all. For each of the examples in (8) and (9) there are two representations which satisfy these requirements (excluding representations containing redundant links). I illustrate in (24) with the example from (9d).

(24) a. If anyone likes John, HE likes him

b. If anyone likes John, HE likes him

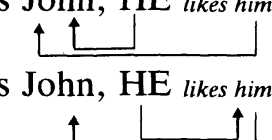

For the example in (20), on the other hand, there is only one such representation:

(25)

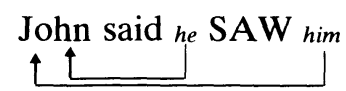

We can account for the distinction between (9d) and (20) by excluding the representation in (25) while allowing for at least one of the representations in (24). This can be accomplished straightforwardly by incorporating into our definition of binding a restriction on co-linking such as that argued for in Higginbotham (1983).

(26) $\alpha$ is bound by $\beta$ if and only if $\beta$ c-commands $\alpha$ and

i: $\quad \alpha$ is linked to $\beta$, or

ii: there is some $\gamma$ such that $\alpha$ and $\beta$ are linked to $\gamma$ and neither $\alpha$ nor $\beta$ is linked to the other.

By the revised definition of bound given in (26), in both (24a) and in (25) the embedded pronoun him is illicitly bound within its binding category by the pronoun he, violating Binding Condition B. Since we have previously eliminated all other potential representations for (20), excluding (25) gives us the desired result that there is no grammatical representation of (20) in which John, he and him are connected by links, and we consequently derive the fact that intended identity is impossible between these three expressions. In contrast, in the case of (9d) we have eliminated one further potential representation in which John, he and him are connected by links - that given in (24a) - though the representation in (24b) remains, leading to the correct prediction 
that intended identity between John, he and him should be possible.

While adopting an asymmetric relation of syntactic identity makes possible a deaccenting based account of the distinction between (9) and (11), when we look at more complex cases in which pronouns and Rexpressions are deaccented together with the predicates which assign them their $\theta$-roles, we find that this single syntactic relation of identity fails to provide the necessary basis for accounting for the full range of examples in need of explanation. As shown in detail in Tancredi $(1992,1993)$, when two or more expressions in a (sub-)sentential domain $D^{8}$ are deaccented, the sentence containing $D$ is only felicitous if the local context in which $D$ occurs contains all of those expressions standing in the same thematic relations that they stand in within $D .{ }^{9}$ Such combinativity of deaccented expressions, as I will call it, can be illustrated with the simple sentence in (27). ${ }^{10}$

(27) MARY likes Bill.

In this sentence, both the verb likes and the object Bill have been deaccented. Mere inclusion of these expressions in the context is not sufficient to make (27) felicitous, as can be seen by considering (27) in each of the two contexts below.

(28) a. Sue doesn't like Bill. However, MARY likes Bill.

b. Bill doesn't like Sue. However, \#MARY likes Bill.

8 In Tancredi (1993), I argue that the relevant domain is the intonation phrase. Here I will remain neutral on the question of how to properly characterize such a domain. In what follows, the precise characterization of such a domain will not play a significant role.

9 As stated, the analysis does not yet allow for the deaccenting in the following examples unless the verbs pleases and likes have the exact same semantic interpretation.

(i) Cecilia likes yogurt. Yogurt pleases Miyuki too.

(ii) Cecilia likes yogurt. Popsicles please Cecilia too.

If we choose to analyze the verbs as differing only in the positions to which they assign their thematic roles, then the deaccenting facts follow directly. On the alternative choice, the felicity of the deaccenting will have to derive from the ease of accommodating an expression containing the predicate-argument combination that is deaccented in the second sentences. (Thanks to an anonymous reviewer for bringing this consequence of the analysis to my attention. Cf. also footnote 12.)

10 The examples I use to illustrate the combinativity of deaccenting could all be analyzed as containing deaccented VPs, obviating the need for anything but standard compositional rules applying within a single constituent. However, as shown in Tancredi $(1992,1993)$, combinativity of deaccenting cannot be restricted to applying to syntactic constituents since a deaccented subject and transitive verb show identical combinativity effects. 
The distinction between (28a) and (28b) can be explained in terms of combinativity of deaccenting since only in the former is the thematic relation between likes and Bill identical in both the context and the target.

When the object is a pronoun, as in (29), the same restriction holds.

(29) MARY likes him.

As with (27), this sentence is only felicitous in contexts which contain a verb-object combination identical to that in the VP likes him. We see from the examples below that the relevant notion of identity is not identity of form, but rather identity of interpretation. (29) is felicitous only in contexts containing an expression of the form like $x$, where the interpretation of the deaccented pronoun him is identical to the interpretation of $x$.

(30) a. John doesn't like him (said pointing to Bill). However, MARY likes him.

b. John doesn't like Bill. However, MARY likes him.

(30a) shows that identity of form is not sufficient, since the deaccented pronoun him in the second sentence cannot differ in its interpretation from the pronoun in the context sentence (i.e., it must be taken as referring to Bill). ${ }^{11}$ (30b) shows that identity of form is not necessary, the antecedent for the pronoun being a name.

We now have all of the pieces in place that we need to argue against a reduction of intended identity to a single, asymmetric syntactic identity relation. An asymmetric syntactic identity relation was seen to make such a reduction plausible on the assumption that a pronoun or $\mathrm{R}$-expression is deaccentable only if linked to some other expression. If we incorporate into this analysis the notion of combinativity of deaccented expressions, we obtain the following:

(31) For a sentence $S$ which contains two expressions $x$ and $y$, where $x$ and $y$ are directly thematically related and $y$ is a pronoun or R-expression, $S$ is felicitous in a context $C$ only if $C$ contains two expressions $x^{\prime}$ and $y^{\prime}$ such that $x^{\prime}$ is identical to $x, x^{\prime}$ stands to $y^{\prime}$ in the same thematic relation

$11 \mathrm{~F} \& \mathrm{M}$ propose similar restrictions in the case of VP deaccenting by requiring that deaccented expressions reconstruct previously occurring expressions. However, their analysis fails to generalize to the full range of cases in which multiple expressions are deaccented within a single sentence since it fails to allow for combination of expressions which are thematically related but which fail to constitute an independent syntactic constituent. 
as $x$ stands to $y$, and $y$ is linked to $y^{\prime} .^{12}$

This can be seen to correctly explain the examples considered in (28) and (30) above by allowing representations parallel to (32a) while excluding those parallel to (32b) below.
a. Sue doesn't like Bill.
$\uparrow$
b. Bill doesn't like Sue. However, \#MARY likes Billhim.

In both examples, the deaccented verb likes is identical to the verb in the context sentence. In (32a), the deaccented object of this verb Bill / him is additionally identical to (or more specifically linked to) the corresponding object of the context sentence, in conformity with (31), and so the sentence containing the deaccented expressions is acceptable. In contrast, the deaccented object in (32b) is not identical with the corresponding object in the context sentence, in violation of (31), and correspondingly the second sentence in the example is unacceptable.

Consider now the following (context initial) example.

(33) John thinks Mary likes him. BILL thinks SUE likes him.

According to (31), the only way for this example to be felicitous is for the pronoun of the second sentence to be linked to that in the first sentence. Linking this latter pronoun to the subject John will result in the representation in (34), giving a strict, or invariant interpretation for the second pronoun.

(34) John thinks Mary LIKES him. BILL thinks SUE likes him.

12 Here and throughout I am assuming that deaccenting is licensed only by identity. There is a fairly large class of felicitous instances of deaccenting which would appear on the surface to be inconsistent with such an analysis, an extreme case based upon an example from Lakoff (1972) illustrated below:

(i) John called Mary a republican, and then BILL insulted her.

Here the VP insulted her has been deaccented, felicitously so, and yet the context does not contain any expression which is identical with this VP. I argued in Tancredi $(1992,1993)$ that such cases of deaccenting force one to increment the local context with some expression containing a VP identical to the deaccented VP, with the content so added constrained by Gricean principles. To do full justice to the analysis here would take me too far afield from the present discussion, so I refer the interested reader to the works cited. For the remainder of this paper I will continue to assume that the relevant licensing condition for deaccenting is true identity, restricting examples to those in which the question of whether such identity obtains can be given a clear and obvious answer. 
The example also admits of a sloppy, or covariant interpretation for the second pronoun. Accounting for this interpretation, however, leads to problems. For F\&M, covariant interpretation of a pronoun derives from the pronoun standing in an identical dependency relation as its antecedent. Translating this into our current notation, this would require the following representation for (33).

(35) John thinks Mary LIKES him. BILL thinks SUE likes him.

However, we have already seen in (32b) that such a representation cannot be allowed, since it violates the combinativity requirements of deaccented expressions in (31). This restriction requires that the second pronoun be linked to the first.

\subsection{From Reference to Bound Variables}

The standard way around this problem is that found in Sag (1976a, 1976b) and Williams (1977) of allowing pronouns in English to be interpreted either as referential-like expressions or as bound variables, and correlating these interpretations with strict and sloppy identity readings respectively. A sloppy reading for (33) could then be generated by raising the names John and Bill and linking the first pronoun not to John but rather to the trace left behind by John as in (36). ${ }^{13}$

(36) John [t thinks Mary LIKES him.] BILL [ $\mathbf{t}^{\prime}$ thinks SUE likes him.]

The linking in the first sentence can plausibly be taken to identify the interpretation of the pronoun with that of the trace $t$ left behind by John, leading to a bound variable like interpretation for that pronoun.

13 Note that F\&M do not adopt this solution. Rather, they take sloppy identity in cases like this to derive from representations in which the pronoun in the antecedent clause is assigned a $\beta$-occurrence of its index. This assumption, however, conflicts with their requirement that deaccented expressions be reconstructions of previously occurring expressions. The deaccenting of the pronoun in the first clause of (33) on this assumption precludes its being analyzed as a dependent expression (i.e. one with a $\beta$ occurrence of an index): R-expressions can never bear $\beta$-occurrences of an index, and F\&M's notion reconstuction imposes identity of indexical type. The only representation available for the first sentence in that example is thus the following:

(i) $\mathrm{John}_{1}{ }^{\alpha}$ thinks Mary likes him $_{1}{ }^{\alpha}$

This representation, however, as F\&M show, only gives rise to a strict reading of the pronoun in the second clause. See section 4.1 for more details. 
To identify the second pronoun with the trace $t^{\prime}$ left behind by $B I L L$, it is necessary for these two expressions to be connected by links, as in (37).

(37) John [t thinks Mary LIKES him.] BILL [ $\mathbf{t}^{\prime}$ thinks SUE likes him.]

This will give rise to a sloppy identity interpretation as desired. Furthermore, every aspect of the representation can be independently shown to be necessary. However, the analysis can now be straightforwardly shown to be incorrect.

Consider the following example from Higginbotham (forthcoming).

(38) Every professor admires HIM.

On current assumptions, the only possible LF representation of (38) which satisfies Conditions $\mathrm{B}$ and $\mathrm{C}$ and in which the pronoun can be interpreted as a bound variable is that given in (39) below.

(39) Every professor [ $t$ admires HIM]

If there were no link connecting the trace $t$ and the pronoun $H I M$, then these two expressions could not be interpreted as identical, and the pronoun could not be interpreted as a bound variable. Furthermore, if $H I M$ were linked to $t$ instead of the other way around, we would have a straightforward Condition $B$ violation. Since the sentence is acceptable on a bound variable interpretation of the pronoun, it follows under our present assumptions that the representation given in (39) must be well-formed.

Consider now what happens when we combine (39) with the analysis of sloppy identity in (37). The conclusion we are led to is clear: If the sentence Every professor admires him is embedded in a context containing a bound variable pronoun, it should be possible to deaccent the pronoun him while still interpreting it as a bound variable. That is, we predict that the deaccented pronoun in the second sentence in (40) below should be interpretable as a variable bound by the quantified expression every professor, with the representation in (41). This prediction, however, is not borne out.

(40) (In this university,) No administrator thinks people admire him. (However,) \#Every professor admires him.

(41) No administrator [ $\mathrm{t}$ thinks people admire him. $]$ Every

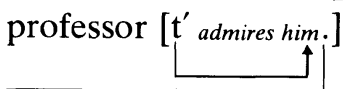


Regardless of the context in which (40) occurs, the deaccented pronoun in the second sentence can never receive a bound variable interpretation. ${ }^{14}$ Since each of the links in (41) is independently required, the single asymmetric identity analysis of intended identity cannot exclude this representation, and hence cannot be the correct analysis.

\section{Dual Identity Syntax}

The data examined in the preceding sections are summarized schematically in (42) through (44), where the first expression is assumed to c-command the second, square brackets indicate the binding domain of the second expression, and all expressions are intended identically.

(42) (Null Context)

(43) $($ Context $=\ldots$ John ...)
a. [JOHN/HE ... JOHN/ HIM]
b. ${ }^{*}[\mathrm{JOHN} / \mathrm{HE} \ldots \mathrm{John} /$ him $]$
a. [JOHN/HE ... John/him $]$
b. [John/he ... JOHN/HIM]
(cf. (9), (14))
c. * [John/he ... John/him $]$

(44)

(Any Context)
a. [QP ... HIM]
b. ${ }^{*}[\mathrm{QP} \ldots \mathrm{him}]$
(cf. (38))
(cf. (33), (40))

If intended identity is to be reduced to syntactic identity, then all of the expressions in each of these examples must be syntactically represented as identical. An analysis which admits only a single syntactic identity relation which is symmetric can account for the pattern of acceptability exhibited in (42) and (43a, b), but cannot account for that in (43c). An analysis in which the single syntactic identity relation is asymmetric fairs better with (43c), but falters when it comes to accounting for the examples in (44). At this point there are two approaches one can take. Like $F \& M$, one could attempt to maintain that there is only one notion of syntactic identity, but constrain this identity relation by two different sub-theories of grammar-Binding Theory and Dependency Theory. Alternatively one could posit two separate syntactic identity relations, each with its own separate properties and constraints.

\footnotetext{
14 On the assumption that the context provided is exhaustive, in the present example the second occurrence of the pronoun him is in fact uninterpretable.
} 
Before developing the latter approach, I first show that the former approach cannot explain the above data.

\subsection{Fiengo and May}

As mentioned earlier, F\&M posit one basic type of syntactic identity relation, represented by coindexing. However, they posit two distinct ways of semantically valuating indexed expressions. An indexed expression can either be independently valuated, in which case its semantic value is given directly via a function assigning referents to indices, or it can be dependently valuated, in which case its semantic value is given indirectly as the value assigned to a coindexed, independently valuated expression. To distinguish these two ways of assigning values to expressions, $F \& M$ categorize occurrences of indices into types $-\alpha$-occurrences (independently valuated), and $\beta$-occurrences (dependently valuated). The two types of valuation are illustrated below.
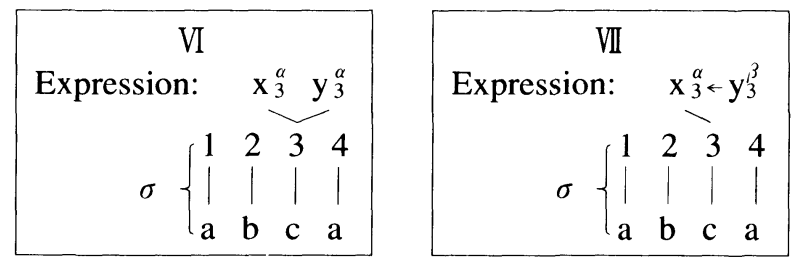

While the distinction between these two types of valuation is important for solving problems involving ellipsis resolution, it is unclear whether F\&M also intend for this distinction to affect the intentions with which a sentence not involving ellipsis can be uttered. Coindexing alone suffices to ensure that two referential expressions will be intended identically, regardless of the types of indices $(\alpha$ or $\beta$ ) employed. Furthermore, if employing indices of different types is to affect the intentions with which a sentence can be uttered, it is far from obvious what this effect could be. One might imagine that the purpose of distinguishing these two types of indices is to capture the intuition that some expressions appear to depend on other expressions for their interpretation while some do not. However, this cannot be the notion of dependence that the proposed distinction is meant to capture since this distinction obtains as much across sentences as within sentences, as seen in (45), whereas formal dependencies involving $\beta$-occurrences of expressions must according to $F \& M$ be resolved within a single 
sentence.

a. John ${ }_{1}^{\alpha}$ thinks I saw $\underline{\operatorname{him}}_{1}{ }^{\alpha} / \underline{\operatorname{him}}_{1}{ }^{\beta}$.

b. $\overline{\mathrm{John}}_{1}{ }^{\alpha}$ is here. I saw him ${ }_{1}^{\alpha / *} \underline{h i m}_{1}{ }^{\beta}$.

It would appear that the formal distinction between index types has no intuitive basis. Furthermore, while only $\beta$-expressions can be used for establishing formal dependency relations, all indexed expressions, regardless of the type of index they bear, are restricted by Binding Theory principles $\mathrm{A}, \mathrm{B}$ and $\mathrm{C} .{ }^{15}$

F\&M do not deal with the effects of focus and deaccenting on the acceptability of intended identity more than superficially. The one proposal that they do make is that for an expression to be deaccented (or deleted) it must be a reconstruction of an expression occurring elsewhere in the discourse. Reconstruction is F\&M's term for "identical in relevant respects." For indexed expressions these relevant respects include identity of indexical type $(\alpha$ or $\beta$ ) plus for expressions that bear $\alpha$-occurrences, identity of indexical value, and for expressions bearing $\beta$-occurrences, either identity of indexical value or resolution in identical dependency relations (with potentially distinct indices). This analysis gives them a straightforward way of accounting for cases of type (42b) as Binding Theory violations, since the deaccenting will only be possible if the deaccented expression bears an $\alpha$-occurrence of the same index that occurs on the preceding focused expression, as illustrated in (46).

(46) $*\left[\mathrm{JOHN} / \mathrm{HE}_{1}{ }^{\alpha}\right.$.. John/him $\left.{ }^{\alpha}{ }^{\alpha}\right]$

However, the analysis cannot account for the possibility of intended identity in cases of type (42a) within the restrictive assumption that intended identity reduces to syntactic identity, since syntactic identity for $F \& M$ always requires sharing of the very indexical values whose distribution is restricted by Binding Theory. To deal with these cases, they are forced to allow intended identity in the absence of syntactic identity as we saw in section 2 , analyzing the two focused expressions as bearing $\alpha$-occurrences of distinct indices.

While F\&M's analysis of examples of the type in (42) is potentially justifiable, their treatment of examples like those in (43) amounts to no more than a denial of the problem such examples pose. Rather than take their analysis to be responsible for such data, they stipulate that

15 For a more detailed summary of Fiengo and May's technical apparatus, cf. Tancredi (1995). 
the data fall outside the purview of Binding Theory, belonging to a supposed mathematical meta-fragment of English in which Condition $\mathrm{C}$ of the Binding Theory (and presumably Condition B as well) fails to apply. Such a stipulation, beyond being inherently unsatisfying, misses an important generalization-that identity relations imposed by deaccenting are syntactically restricted, while those not so imposed are not. The facts in $(43 a, b)$ could be captured within F\&M's approach by allowing expressions to bear occurrences of multiple indices, as outlined earlier. However, data of the type in (43c) would remain problematic.

I argued above that data like (43c) could be explained using an asymmetric identity relation by analyzing the deaccented expressions as both dependent upon the same expression in the context and requiring that such co-dependent expressions satisfy Binding Conditions with respect to one another. This analysis, however, is not available to F\&M. The only asymmetric identity relation they admit is a sentence internal dependency relation, where both the dependent expression and its antecedent are required to be constituents of the same phrase marker. The pattern observed in (43c), however, obtains not only when the contextual occurrence of the antecedent is within the same sentence as the deaccented expressions as in (14) but also when they are in separate sentences, as in (47) below.

(47) A: Does anyone like John?

B: I don't know. However, JOHN $/ \mathrm{HE} /{ }^{*}{ }_{\text {John }} /{ }^{*}{ }_{h e}$ HATES John $/$ him.

Furthermore, adopting the solution offered above would require treating all deaccented expressions as dependent expressions, whereas F\&M explicitly deny that names can be dependent. The changes required to fix these two problems are so fundamental that the theory that would result from making the changes could hardly be seen as a variant of the original.

The final class of problems given in (44) is particularly problematic for $F \& M$. Bound variable interpretation of a pronoun for $F \& M$ requires coindexing of the pronoun with the variable left behind by raising of the binding expression. Since such coindexing is on their analysis restricted by Condition B of the Binding Theory regardless of the type of occurrence of the indices involved, binding of the pronoun in (44) should be impossible regardless of whether or not the pronoun is focused, contrary to fact. This case contrasts with the case of (42a), 
since coindexing of the pronoun and the trace of the QP in (44) is required for a bound variable interpretation of the pronoun regardless of what other types of intentions one has or fails to have in uttering such a sentence, whereas coindexing can be argued not to be required for the looser notion of mere intended identity in (42a). The inability to handle the full paradigm of examples in (42) through (44) shows F\&M's analysis to be inadequate.

\subsection{An Alternative Analysis}

It is clear from the summary of data given in (42) through (44) that a distinction needs to be made between focused expressions and deaccented expressions. Two generalizations can be made from these examples. The first is that a Binding Theory type of violation occurs only when the "bound" expression is deaccented, as in (42b), (43d), and (44b), and the second is that such a violation results only when the deaccented expression cannot escape being identified with an illicit antecedent. In (42b) this is the case since deaccenting forces identity with some preceding expression and the only such expression available is the c-commanding subject within the deaccented expression's binding domain. In (43d) this will also be so on the assumption that any two deaccented expressions which are identified with the same antecedent will themselves necessarily be identical. Since the context contains only a single occurrence of John, identification of each of the deaccented expressions with this occurrence of John will thus make it impossible for the two expressions to escape being identified with each other. Finally, in (44b), while deaccenting of the pronoun can be licensed by the occurrence of a (bound variable) pronoun in the previous context, the deaccented pronoun still must be identified with the trace bound by the quantified expression (at LF) in order to be interpreted as a variable bound by that expression.

These informal comments together with the remainder of the data put some clear constraints on the form that an analysis of the facts must take:

(48) The identity relation required to hold between a deaccented expression and some other expression in the preceding context is the same as the identity relation which is constrained by Binding Theory.

(49) Focus licenses an alternative means for identifying expressions, one which is not constrained by Binding Theory, 
and which is not available for deaccented expressions.

Finally, from the distinction found in (44) together with standard cases of long distance binding of a pronoun by a quantified antecedent in which the pronoun is typically deaccented, it would appear that the following holds as well:

(50) Either type of identity relation is capable of giving rise to a bound variable interpretation of a pronoun.

While there are several conceivable ways of satisfying these constraints and hence of accounting for the data under consideration, for concreteness I propose the following analysis, predicated on one symmetric identity relation (represented by coindexing) and one asymmetric identity relation (represented by linking).

I: An expression $x$ is i-bound if and only if there is some expression $y$ such that $x$ and $y$ are co-indexed and $y$ c-commands $x$. An expression which is not $\mathrm{i}$-bound is i-free.

II: An R-expression or pronoun $x$ can be deaccented only if it is coindexed with some other expression $y$ contained in the context preceding $x$.

III: An R-expression or pronoun $x$ can be linked to some other expression $y$ only if $x$ is focused.

IV: Condition B: A pronoun must be i-free in its binding domain.

V: Condition C: An R-expression must be i-free everywhere.

VI: A pronoun $p$ is interpreted as a variable bound by a (quantified) expression $Q$ if and only if $p$ is c-commanded by $Q$ at LF, and

i: $\quad p$ is linked to the trace left by raising $Q$; or

ii: $p$ is coindexed with the trace left by raising $Q$.

This analysis accounts for the examples illustrated schematically in (42) through (44) by making possible only the following (LF) representations, of which those excluded by Condition $\mathrm{B}$ or $\mathrm{C}$ of the Binding Theory are marked with an asterisk $\left({ }^{*}\right)$. (The arrow pointing to the left in the representations in (52a-ii) and (52b-ii) is intended as a link to the occurrence of $J o h n_{\mathrm{i}}$ in the context.)

(51) (Null Context)
a. i: ${ }^{*}\left[\mathrm{JOHN} / \mathrm{HE}_{\mathrm{i}} \ldots \mathrm{JOHN} / \mathrm{HIM}_{\mathrm{i}}\right]$
ii: $[\mathrm{JOHN} / \mathrm{HE} \ldots \mathrm{JOHN} / \mathrm{HIM}]$
b. ${ }^{*}\left[\mathrm{JOHN} / \mathrm{HE}_{\mathrm{i}}\right.$... John/him $]$

(52) $\left(\right.$ Context $\left.=\ldots \operatorname{John}_{\mathrm{i}} \ldots\right)$

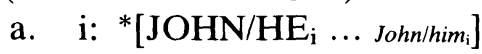




\author{
ii: $[\mathrm{JOHN} / \mathrm{HE}$... John/him $\mathrm{i}]$ \\ b. i: * [John/he $\ldots$ JOHN/HIM \\ ii: $\left[J o h n / h e_{\mathrm{i}} \ldots J \mathrm{JOHN} / \mathrm{HIM}\right]$ \\ iii: $\left[\begin{array}{llll}\text { John/he } & \ldots & \text { JOHN/HIM] }\end{array}\right.$ \\ c. ${ }^{*}\left[\begin{array}{llll}J^{\prime} o h n / h e_{\mathrm{i}} & \text {. . John/him }\end{array}\right]$ \\ (53) (Any Context)
a. i: *EVERY PROFESSOR $\left[\mathrm{t}_{\mathrm{i}} \ldots \mathrm{HIM}_{\mathrm{i}}\right]$
ii: EVERY PROFESSOR [t ... HIM] \\ b. *EVERY PROFESSOR $\left[\mathrm{t}_{\mathrm{i}} \ldots h{ }^{\text {nim }} \mathrm{i}\right]$
}

\title{
5. Consequences and Applications
}

The most significant consequence of the above analysis is that it makes it possible to reduce intended identity to syntactic identity. We have seen this for a fair range of examples, including typical examples of intended identity between pronouns and $\mathrm{R}$ expressions and that involved in bound variable interpretations of pronouns. There remains one further case of intended identity which has yet to be addressed, that involved in identity statements. From the following examples, however, it is clear that these fit into the analysis outlined above without further stipulation.

$$
\begin{array}{lll}
\text { a. i: THAT MAN is JOHN. } & \text { ii: *THAT MAN is John. } \\
\text { b. i: } \text { JOHN is HIM. } & \text { ii: * }{ }^{*} \text { JOHN is him. }
\end{array}
$$

As context initial utterances, only the examples in (i), in which the post-copular occurrence of John / him is focused, are acceptable. Since focused expressions on the analysis from section 4.2 can be identified with a preceding expression through linking, and since linking is not constrained by Binding Conditions, these examples are predicted to allow of representations in which the second underlined expression is linked to the first. The situation changes drastically if the post-copular expressions are deaccented as in (ii), and again the result is just what is predicted by the theory. As context initial utterances, the only potential antecedents for the deaccented expressions are the subjects of their respective sentences. The co-indexation required by the deaccenting, however, will yield a straightforward Condition $\mathrm{B} / \mathrm{C}$ violation, and thus the sentences are predicted to be unacceptable, as 
indeed they are. Note in passing that without an adequate analysis of the interaction between focus, deaccenting and intended identity, F\&M's original analysis of identity statements fails to adequately distinguish the acceptable examples in (i) from the unacceptable examples in (ii). In all cases, the copula functions similarly to predicate identity between its subject and object, and hence should according to F\&M's analysis license an intended identity reading in all four cases in (54).

In addition to making possible a reduction of intended identity to syntactic identity, the analysis furthermore extends the range of data which can be accounted for in a principled fashion by formalizing aspects of the relation between sentences and discourse contexts. Each of the formal notions of syntactic identity can obtain either between expressions within a single sentence or between expressions in distinct sentences, as is clear from the analysis given in (52) for the examples in (43). By bridging sentence internal syntax and what could be called inter-sentential syntax in this fashion, it becomes possible-at least in a limited fashion-to predict some of the effects that a discourse context will have on acceptability.

As an illustration of the kinds of benefits which can result from this type of marriage of sentence internal and sentence external syntax, consider the following paradigm, all examples intended context initially.

(55) a. John went to see "Jaws". Then, BILL went to see a movie.

b. John went to see a movie. Then, \#BILL went to see "Jaws".

c. John Smith's mother LIKES him.

d. (pointing) \#HIS mother LIKES John Smith. ${ }^{16}$

In the examples in $(55 a, b)$ we see that deaccenting of a less specific

16 This same sentence has often been claimed to be acceptable, in opposition to the following sentence which differs only in its focus structure.

(i) His mother likes JOHN SMITH.

While there is clearly a difference between these sentences, however, I am loath to attribute these differences to binding distinctions, especially given the fact that neither of the relevant expressions c-commands the other. Note that the acceptability of (55d) differs depending on whether a context is supplied (or accommodated) containing mention of John Smith. For example, the following discourse is perfectly acceptable with his and John Smith intended identically throughout.

(ii) A: Tell me something about John Smith.

B: Well, for one thing, his mother LIKES John Smith.

On the present analysis, this behavior is expected. In (ii), both his and John Smith 
expression in the context of a more specific expression is acceptable, while the reverse is not. The phenomenon in question is clearly a discourse phenomenon in that the expressions being compared occur in separate sentences. However, by analyzing these same discourse processes as occurring within a sentence, the otherwise puzzling array of restrictions on identity brought out clearly in Lasnik (1989) and illustrated in $(55 \mathrm{c}, \mathrm{d})$ can be handled straightforwardly in the exact same way as the distinction between $(55 a, b)$ is handled. Here, deaccenting of the less specific expression him can be licensed by the more specific John Smith, while deaccenting of the latter licensed by the former is impossible.

If the above comments are on the right track, they indicate that a new division of labor is in order between a purely sentence internal binding theory and an inter- and intra-sentential theory of discourse deaccenting. From the discussion of examples such as (44), it appears that at least some vestige of binding theory must remain intact in order to handle restrictions on bound variable pronouns which are independent of restrictions on deaccenting. Beyond this, however, the question remains open of where to draw the line between Binding Constraints and Deaccenting Constraints in accounting for data traditionally considered to lie exclusively in the realm of Binding Theory.

In developing an analysis of the facts, F\&M's analysis has been

within B's response can be legitimately deaccented, each licensed by the occurrence of John Smith in A's request. The resulting identity between the two expressions is in conformity with Binding Conditions, and so no unacceptability is predicted. The more problematic case under the present analysis is that of (i). As the analysis stands, it predicts that intended identity between his and John Smith should be possible even context initially, since John Smith is focused and hence should be able to be linked to his. Such intended identity does not appear to be possible. We can overcome this problem if we assume that linking is a discourse process constrained in (some of) the same ways that deaccenting is constrained. In particular we could assume that linking to a less specific expression is illicit much as deaccenting licensed by a less specific expression is illicit. Such an extension of the present analysis would make it possible not only to explain the contrasts found in (55d), (i) and (ii), but would furthermore correctly predict the acceptability of (iii) as a context initial sentence.

(iii) John Smith's mother likes JOHN SMITH.

Whether this extension can be worked out in detail remains to be shown, though the approach looks promising. 
shown to be inadequate, requiring at the very least to be supplemented if a deterministic connection between syntactic identity and implicitly intended identity is to be forged. The analysis arrived at in section 4.2 explains all of the core facts that F\&M swept under their mathematical meta-fragment carpet, making such a connection look plausible. This suggests that F\&M's proposed meta-fragment should be abandoned and replaced by that analysis. Adopting this amendment makes it unnecessary to supplement the syntactic analysis with a pragmatic analysis for any of the cases considered here, and hence the resulting analysis can be counted as a truly syntactic analysis of implicit intended identity. Whether the analysis can be maintained in whole or only in part I leave as a question for future research.

\section{REFERENCES}

Chomsky, Noam (1976) "Conditions on Rules of Grammar," Linguistic Analysis 2, 303-351.

Chomsky, Noam (1980) "On Binding," Linguistic Inquiry 11, 1-46.

Chomsky, Noam (1981) Lectures on Government and Binding, Foris, Dordrecht.

Evans, Gareth (1977) "Pronouns, Quantifiers, and Relative Clauses," Canadian Journal of Philosophy 7, 467-536.

Evans, Gareth (1980) "Pronouns," Linguistic Inquiry 11, 337-362.

Fiengo, Robert and Robert May (1994) Indices and Identity, MIT Press, Cambridge, MA.

Higginbotham, James (1983) "Logical Form, Binding, and Nominals," Linguistic Inquiry 14, 395-420.

Higginbotham, James (forthcoming) "Anaphoric Reference and Common Reference," Linguistic Inquiry.

Lakoff, George (1968) "Pronouns and Reference," Indiana University Linguistics Club, Bloomington, Indiana.

Lakoff, George (1972) "Linguistics and Natural Logic," Semantics of Natural Language, ed. by Donald Davidson and Gil Harman, 545-665, Reidel, Dordrecht.

Lasnik, Howard (1976) "Remarks on Coreference," Linguistic Analysis 2, $1-22$.

Lasnik, Howard (1989) "On the Necessity of Binding Conditions," Principles and Parameters in Comparative Grammar, ed. by Robert Freidin, 7-28, MIT Press, Cambridge, MA.

Pierrehumbert, Janet (1980) The Phonology and Phonetics of English Intonation, Doctoral dissertation, MIT. 
Reinhart, Tanya (1983) Anaphora and Semantic Interpretation, University of Chicago Press, Chicago.

Sag, Ivan (1976a) Deletion and Logical Form, Doctoral dissertation, MIT.

Sag, Ivan (1976b) "A Logical Theory of Verb Phrase Deletion," CLS 12, 533-547.

Selkirk, Lisa (1984) Phonology and Syntax, MIT Press, Cambridge, MA.

Tancredi, Christopher (1992) Deletion, Deaccenting and Presupposition, Doctoral dissertation, MIT.

Tancredi, Christopher (1993) "Focus-Related Felicity," ms., Yokohama National University.

Tancredi, Christopher (1995) "Review of Fiengo and May's Indices and Identity," Studies in English Literature, English Volume, 136-147.

Williams, Edwin (1977) "Discourse and Logical Form," Linguistic Inquiry 8, 101-139.

7-32-4 Shirane, B-103

Asahi-ku, Yokohama-shi

Kanagawa 241

e-mail: tancredi@ed.ynu.ac.jp 